\title{
Calidad de la escritura académica: comparación de los efectos de comentarios de pares de escritura y expertos
}

\author{
Yudi Herrera-Núñez
}

\begin{abstract}
Resumen
Los comentarios de revisión de los pares se pueden convertir en una estrategia para influir en la calidad de la escritura de los estudiantes. Este estudio propone identificar el grado de influencia de los comentarios de revisión de escritores experimentados y pares en la escritura del texto final. La metodología adoptada da cuenta del tipo de comentarios de escritores expertos y pares de escritura según la taxonomía de revisiones de Faigley y Witte (1981), y el análisis temático sobre el tipo de comentarios de revisión y la escritura reflexiva. La muestra estuvo conformada por 50 textos finales, dos borradores y las reflexiones de escritura de 50 estudiantes del curso de Comunicación Académica de una universidad pública chilena. Entre las principales conclusiones podemos señalar que tanto la recepción como la elaboración de comentarios inciden en la mejora de la calidad de la escritura y generan actitudes más positivas hacia la escritura. En general, los estudiantes expertos producen más comentarios de profundidad, aunque menos elogiosos y al participar de actividades de revisión, los estudiantes toman conciencia de su propio proceso (referencia, estructuración de párrafos, adición de información, parafraseo y síntesis) como en la adopción de estrategias de escritura al ofrecer comentarios formativos a sus pares.
\end{abstract}

Palabras clave:

comentarios de revisión; revisión por pares; escritura académica. 


\section{Qualidade da escrita académica: comparar os efeitos dos comentários de colegas e peritos}

Resumo: Os comentários de revisão pelos pares podem tornar-se uma estratégia para influenciar a qualidade da escrita dos estudantes. Este estudo propõe identificar o grau de influência dos comentários de revisão de pares e escritores mais experientes na redacção do texto final. A metodologia adoptada dá conta do tipo de comentários de escritores pares e escritores mais experientes, segundo a taxonomia de revisão de Faigley e Witte (1981), e a análise temática sobre o tipo de comentários de revisão e escrita reflexiva. A amostra consistiu em 50 textos finais, dois esboços, e as reflexões escritas de 50 estudantes do curso de Comunicação Académica de uma universidade pública chilena. Entre as principais conclusões podemos salientar que tanto a recepção como a elaboração de comentários têm um impacto na melhoria da qualidade da escrita e geram atitudes mais positivas em relação à escrita. Em geral, os estudantes especializados produzem comentários mais profundos, sendo embora menos elogiosos, e pela sua participação em actividades de revisão, os estudantes tomam consciência do seu próprio processo (referência, estruturação de parágrafos, adição de informação, paráfrases e síntese), como também na adopção de estratégias de escrita, oferecendo comentários formativos aos seus pares.

Palavras-chave: comentários de revisão; revisão por pares; escrita acadêmica.

\section{Quality of academic writing: comparing the effects of comments from writing peers and experts}

Summary: Peer review comments can become a strategy to influence the quality of student writing. This study proposes to identify the degree of influence of the review comments of experienced writers and peers in the writing of the final text. The methodology adopted compares the type of comments of expert writers and writing pairs according to the taxonomy of reviews by Faigley and Witte (1981), and the thematic analysis on the type of review comments and reflexive writing. The sample consisted of 50 final texts, two drafts and the writing reflections of 50 students of the Academic Communication course of a Chilean public university. Among the main conclusions, we can point out that both the reception and the elaboration of comments affect the improvement of the writing quality and generate positive attitudes towards writing. In general, the expert students produce more in-depth comments, although less complimentary, and and when the students participate in review activities, they become aware of their own writing process (reference, paragraph structure, addition of information, paraphrasing and synthesis). At the same time, they adopt writing strategies by offering formative comments to their peers.

Keywords: review comments; peer review; academic writing.

\section{La qualité de la rédaction universitaire: comparaison des effets des commentaires des pairs rédacteurs et des experts}

Résumé: Les commentaires des pairs peuvent devenir une stratégie pour influencer la qualité de la rédaction des élèves. Cette étude propose d'identifier le degré d'influence des commentaires de révision d'écrivains expérimentés et de pairs dans la rédaction du texte final. La méthodologie adoptée compare le type de commentaires d'écrivains experts et de couples d'écriture selon la taxonomie des revues de Faigley et Witte (1981) et l'analyse thématique sur le type de commentaires de revue et d'écriture réflexive. L'échantillon comprenait 50 textes finaux, deux projets et les réflexions écrites de 50 étudiants du cours de communication académique d'une université publique chilienne. Parmi les principales conclusions, nous pouvons souligner que la réception et l'élaboration de commentaires ont une incidence sur l'amélioration de la qualité de l'écriture et génèrent des attitudes plus positives à l'égard de l'écriture. En général, les étudiants experts produisent des commentaires plus approfondis, mais moins complémentaires, et lorsqu'ils prennent part à des activités de révision, ils prennent conscience de leur propre processus d'écriture (référence, structure de paragraphe, ajout d'informations, paraphrasage et synthèse). Dans le même temps, lls adoptent des stratégies d'écriture en offrant des commentaires formatifs à leurs pairs.

Mots-clés: commentaires de revue; examen par les pairs; l'écriture académique. 


\section{Introducción}

Este artículo se enmarca en la perspectiva sociocultural de la 'literacidad' (Barton, Ivanič, Appleby, Hodge y Tustin, 2012) y desde el enfoque de la pedagogía de la escritura de comentarios escritos de revisión (Shah, Rajgor y Pietrobon, 2010) como mecanismos de aprendizaje (Clark, 2012). Las tareas de escritura y el tipo de comentarios de revisión de la escritura que realizan los docentes, influyen en la manera en la que los estudiantes abordan este proceso.

Los comentarios de revisión de escritura no sólo hacen referencia a la información proporcionada por un agente (docente o par) sobre el desempeño en una tarea, sino que contemplan también, la comprensión que realiza el estudiante sobre la tarea y el propósito de esta, la capacidad de aprender de los comentarios de revisión y su potencialidad de aplicación en posteriores tareas. Hattie y Timperley (2007) citan la síntesis que Winne and Butler (1994) realiza respecto a los ámbitos que entraña esta actividad "feedback is information with which a learner can confirm, add to, overwrite, tune, or restructure information in memory, whether that information is domain knowledge, meta-cognitive knowledge, beliefs about self and tasks, or cognitive tactics and strategies" (p. 82).

A pesar de la nutrida cantidad de literatura sobre los comentarios de revisión, los resultados respecto a su efectividad no son concluyentes. Una parte de la investigación sobre los comentarios de revisión de tareas de escritura ha señalado su ineficacia (Hyland, 2000) haciendo referencia a su imprecisión y falta de claridad (Semke, 1984); mientras que otros estudios evidencian la influencia de cierto tipo de comentarios en la calidad de las tareas de escritura (Lachner, Backfisch y Nuckles, 2018; Min, 2005 y Semke, 1984). La efectividad del feedback ha sido estudiada según sea proporcionado por el docente (Cho y MacArthur, 2011) o por estudiantes pares de escritura (Baker, 2016; Hattie y Timperley, 2007 y Luaces, Diez, y Bahamonde, 2018). Dada la escasez de estudios que abordan el proceso de negociación de los comentarios surge este estudio que pretende abordar el grado de influencia que evidencian en el texto final, los comentarios de las revisiones de proceso (borradores) de los escritores experimentados y de los pares en escritura.

El uso de borradores múltiples de escritura académica es quizá la característica que identifica la evaluación formativa en este ámbito cuyo propósito está en "to provide rich feedback and support for learning (Black \& Wiliam, 1998), and renewed interest in peer assessment as a tool for learning." (Gielen, Peeters, Dochy, Onghena y Struyven, 2010, pág. 304). En lugar de requerir que los estudiantes intenten expresar sus ideas perfectamente en el primer intento, el enfoque de evaluación formativa permite a los estudiantes recibir comentarios tanto de un escritor experimentado como de sus 
pares, para revisar y reescribir al menos una o dos veces su texto, de manera que puedan presentar una "versión final" mejorada (Sommers, 2006).

Los resultados de las investigaciones sobre el uso de múltiples borradores reconocen que la revisión de los pares ayuda a los estudiantes universitarios a obtener más información sobre su escritura y sobre los procesos de revisión, fomentan un sentido de apropiación del texto (Philippakos y MacArthur, 2016), generan actitudes más positivas hacia la escritura (Tsui y Ng, 2000; Zhang, 1995) y mejoran la consideración de adaptar el escrito a una audiencia posible (Zhu, 2018).

\section{Revisión de Borradores de escritura por pares}

La revisión de borradores realizada por pares proporciona oportunidades para el aprendizaje mutuo de la escritura académica, debido a que los revisores y escritores varían en sus fortalezas, modos de expresión y niveles de competencia, por lo tanto, pueden proporcionarse una mutua asistencia. Los resultados del estudio de Lundstrom y Baker 2009) encontraron que los revisores obtuvieron más progresos en la calidad de su escritura que sus compañeros objeto de la revisión.

En lo que respecta a las teorías retóricas, aquellos que subrayan la naturaleza social de la escritura también han favorecido el uso de revisiones por pares (Mandala et al. 2018). La evaluación colaborativa entendida como una negociación compartida tanto de los objetivos como de lo que será evaluado, forma parte del estudio de Dochy, Segers y Sluijsmans (1999) cuyos resultados señalan que los estudiantes involucrados mostraron tener una percepción realista de sus propias habilidades y ser capaces de realizar juicios racionales de sus logros y el de sus compañeros. Mendonça et al. (1994) sostienen que la escritura se deriva de la "conversación" entre los escritores en su comunidad discursiva y que el ambiente colaborativo creado en los grupos de pares es propicio para abordar los problemas de redacción en profundidad entre los escritores, tales como el enfoque y el desarrollo de las ideas.

\section{Modelos de comentarios de revisión}

Modelo de Hattie de revisión por pares, está basado en el metanálisis de investigaciones referidas a la efectividad de las retroalimentaciones, Hattie y Timperley (2007) resumen las características de la retroalimentación efectiva. De acuerdo a su modelo una buena retroalimentación, les dice a los alumnos: (a) 'a dónde van' mediante el explicitación de los criterios del desempeño deseado (b) 'cómo se encuentran' al retroalimentar el desempeño y (c) 'dónde ir a continuación' avanzando hacia la autorregulación del aprendizaje o la ampliación del mismo. El modelo discrimina entre cuatro niveles de retroalimentación: dirigido a la tarea, al proceso de resolución de la tarea, 
dirigidas a desarrollar una competencia autorreguladora y orientada a la valoración de la persona (retroalimentación positiva o elogiosa). Este es uno de los modelos más completos y orientados al aprendizaje.

El modelo de Sluijsmans et al. (2004) desglosa la provisión de retroalimentación entre pares en tres partes: (1) formula discrepancias entre el criterio de logro y el desempeño del par, (2) formula puntos para mejorar, y (3) Reflexiona sobre los puntos de mejora para el par. Se debe considerar que los estudiantes, al participar en la evaluación de pares y el intercambio de opiniones, no deben posicionarse como expertos o como profesores, sino participar en la tarea para aprender activamente.

Min (2005) Ilevó a cabo un estudio en el aula para capacitar a 18 estudiantes como revisores de escritura de segundo año en la clase de inglés como lengua extranjera. Ella identificó cuatro características de los comentarios efectivos para facilitar las revisiones de los estudiantes producto de la revisión bibliográfica sobre esta temática: aclarar las intenciones de los escritores, identificar problemas, explicar la problemática, y hacer sugerencias específicas, y las utilizó como directrices durante el entrenamiento en la clase.

El Modelo GEAR-ed de Huang (2018), es uno de los más actuales, pues incorpora los aportes de los modelos anteriores incluyendo componentes deseables en la retroalimentación entre pares. Este modelo contempla tanto aspectos relacionados con la tarea, así como factores afectivos relacionados con la recepción de los comentarios de revisión. Este modelo se explica, en seguida, a partir de sus iniciales en inglés. En cuanto a la tarea, los componentes son:

G: identificación del problema de escritura entre la meta de aprendizaje y el desempeño actual. E - Explicación del elemento a mejorar. A - Posibilidad de mejora y sugerencias. R - Recursividad, identificar y relacionar elementos de su propio texto que puedan servir al estudiante como fuente o peldaño para propiciar la mejora.

En cuanto a los elementos afectivos, los componentes son: e - elementos cognitivos o afectivos para mostrar o infundir ánimo al estudiante par, $\mathrm{d}$ - expresar de manera cuidadosa y ponderada comentarios de revisión. Aunque no siempre están presentes todos estos criterios, la presencia de los cuatro primeros puede asegurar mayor efectividad.

La literatura revisada revela un foco de atención convergente respecto del efecto de los comentarios de escritura de pares a nivel de la calidad de la escritura, en la negociación de las tareas, al efecto emotivo, al tipo de comentarios y en la percepción de conocimiento experto o par de quien los emite. Al mismo tiempo, se observa una reducida atención al rol del diálogo en el proceso de revisión de escritura (Zhu, 2018). En este contexto, emerge nuestro propósito, comparar los efectos de la calidad de los comentarios de escritura y del proceso de negociación de los mismos en la revisión de borradores múltiples realizado por escritores expertos y estudiantes pares. 
A pesar del consenso general en la comunidad de alfabetización acerca de la escritura de proceso entendida como una experiencia iterativa, recursiva y compleja; en oposición a una secuencia lineal de pasos que los escritores deben seguir para lograr un producto; no compartimos completamente las prácticas habituales sobre los métodos de evaluación de la escritura de proceso. Algunos de los procesos de avance en la escritura no se evidencian suficientemente en las rúbricas empleadas. En el caso de los hallazgos del programa de evaluación de portafolios del Distrito Escolar de Pittsburgh (Arts Propel), en lugar de emplear rúbricas se promueve que el "uso de los procesos y estrategias de escritura" de los estudiantes, se evalúa con una escala de seis niveles. Se les pide a los evaluadores que consideren el uso eficaz de las estrategias de pre-escritura, el uso de borradores para descubrir y dar forma a las ideas, el uso de oportunidades de socialización para refinar la escritura (compañeros, lectores adultos) y el uso efectivo de la revisión (reformulación, reenfoque y modificaciones) (LeMahieu, Eresh, y Wallace, 1992). Los estudios técnicos han demostrado que las evaluaciones de Pittsburgh alcanzan niveles satisfactorios al calificar los procesos y el uso de estrategias de escritura de los estudiantes.

\section{Metodología}

Los participantes en este estudio fueron 50 estudiantes de primer año de una universidad pública en Chile, quienes participan del curso para nivelar habilidades de comunicación académica. Para el análisis del corpus se revisan en el trabajo de escritura de 50 estudiantes, a) dos borradores del proceso de elaboración de un informe de investigación, b) una rúbrica de comentarios de pares y c) los comentarios de revisión del escritor experimentado.

\section{El procedimiento ocurre de la siguiente manera:}

1. Asignación de la tarea de escritura (Definición de los parámetros, negociación de la finalidad y planificación)

2. Primer Borrador (revisión por pares)

3. Segundo borrador (revisión de escritor experto)

4. Texto final

La metodología de análisis se basa en el análisis de contenido de los informes y un texto de reflexión sobre el aprendizaje.

A continuación, se presentan las categorías que nos permitieron evaluar los progresos en la escritura de los borradores múltiples y los indicadores para abordar la reflexión crítica. 


\section{Categorías de análisis respecto de la mejora de escritura de borradores}

Para realizar el análisis de las modificaciones de los borradores respecto de los comentarios de los pares y escritor experimentado, siguiendo el modelo de análisis propuesto por Min (2005), Faigley y Witti (1981) se verificaron este tipo de cambios en los borradores:

\section{Modificaciones Profundas:}

- Adición: el revisor recomienda agregar información

- Eliminación: el revisor recomienda eliminar la información

- Sustitución: el revisor recomienda sustituir información

- Reformulación: el revisor recomienda reescribir la misma (sinónimos, precisar conceptos, entre otros).

- Consolidación: el revisor sugiere integrar información separada unificándola.

- Reestructuración (el revisor sugiere mover información)

\section{Modificaciones superficiales}

- Estilo de escritura el revisor recomienda sujetarse a los rasgos del discurso académico (impersonal y formal)

- Forma Sugiere revisar la ortografía o puntuación.

\section{Discusión y resultados}

Dimensiones revisadas y modificadas en los borradores y la influencia de las revisiones del escritor experimentado y pares.

Nuestro estudio pretende abordar el grado de influencia que evidencian en el texto final, los comentarios de las revisiones de proceso (borradores) de los escritores experimentados en escritura y de los pares de curso. Los escritores experimentados son estudiantes que guían los cursos de escritura académica, revisan y realizan comentarios a los trabajos de escritura de los estudiantes para orientar nuevas versiones de los textos (en este caso un informe). En primer término, nos referiremos al tipo de comentarios de revisión realizados por escritores experimentados y novatos, como se muestra en la siguiente figura: 
Figura 1. Porcentaje de tipo de comentario según revisor.

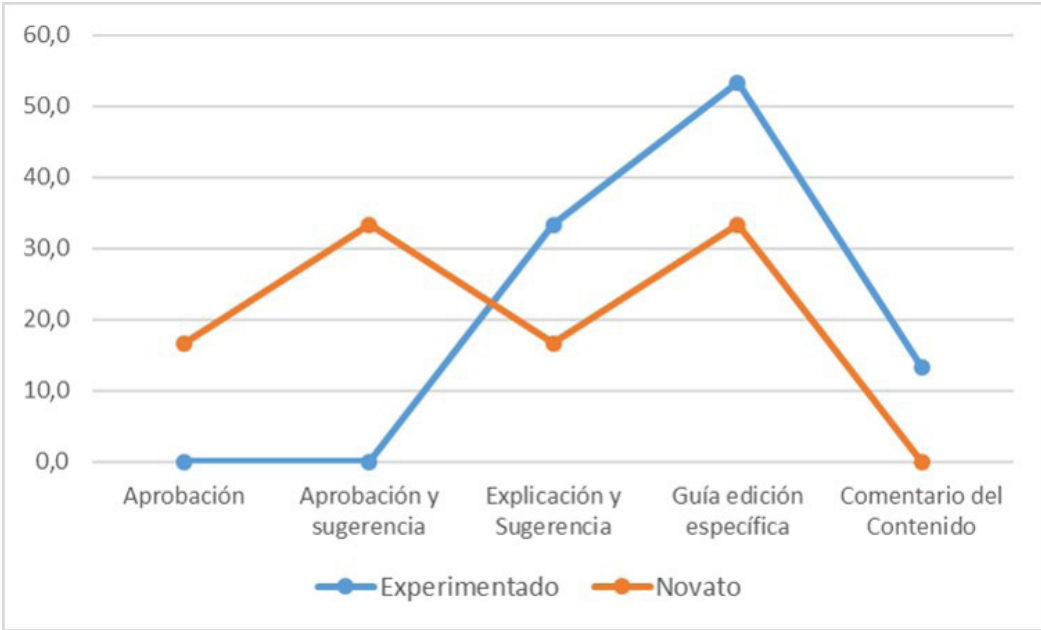

Fuente: Elaboración propia

En la Figura 1. se muestra el porcentaje del tipo de comentario de acuerdo al revisor. Los comentarios afectivos sobre la tarea son realizados sólo por el escritor par en un $16 \%(n=30)$ y con sugerencias en un $33 \%(n=60)$.

Mientras que los comentarios respecto de la tarea son realizados tanto por pares como por los escritores experimentados. Los comentarios más frecuentes se refieren a problemas específicos de edición tanto del estudiante experimentado 53\% ( $n=240)$ como del par novato $(n=60)$. En el caso de las explicaciones y sugerencias los estudiantes experimentados las realizan en un 33\% $(n=150)$ mientras que los estudiantes pares en un 16,6\% ( $n=30)$. Los resultados del estudio de Gielen, Peeters, Dochy, Onghena y Struyven (2010) concuerda con estos datos, al señalar que los escritores expertos brindan más ideas y explicaciones más extensas y, por lo general, incluyen menos elogios, mientras que los comentarios de los compañeros solicitan más detalles.

De acuerdo con estos resultados el perfil de revisor del estudiante par es a la vez afectivo y centrado en la tarea; mientras que el del estudiante experto se centra solo en la tarea.

La revisión como aprendizaje se comprende como un proceso de resolución de problemas que consiste en diagnosticar los problemas del texto, justificar los propios comentarios y generar soluciones, explicándolas para mejorar los textos. En este proceso de revisión, los estudiantes pueden desarrollar estrategias importantes para escribir y mejorar su propia escritura. La generación de explicaciones permite a los estudiantes aclarar la comprensión del contenido y de sus habilidades de escritura para clarificarlas a otros al tiempo que sugieren posibles soluciones (Cho y MacArthur, 2011). 
Respecto de los comentarios referidos a la tarea de escritura, Baker (2016) afirma que los cambios a nivel de superficie permiten conservar el significado original del texto, pero modifican o aclaran la escritura, incluidas correcciones ortográficas y gramaticales, adiciones y eliminaciones de palabras y frases, y sustituciones de palabras. Los cambios a nivel de significado, profundos son aquellos que alteran el significado del texto de manera sustancial. Incluyen agregar matices o una nueva dirección al análisis, agregar nuevos subpuntos o elaboraciones a los argumentos, y revisar los subpuntos o el argumento general del documento. Ambos tipos de cambios son importantes para facilitar la buena escritura.

En ese contexto, se analizan los comentarios de revisión que los escritores experimentados hacen al primer borrador respecto de la tarea de escritura y, se identifica qué tipo de modificaciones se solicita realizar a los estudiantes como se muestra en la Figura 2.

Figura 2. Porcentaje tipo de comentarios de revisión de Escritores Expertos

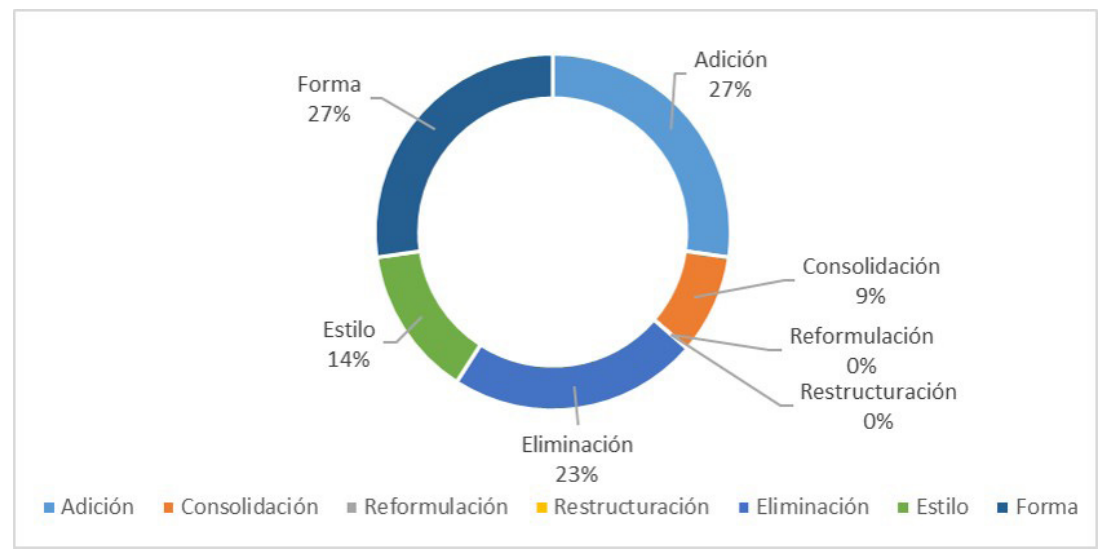

Fuente: Elaboración propia

En la Fig. 2, puede observarse que mayoritariamente, se solicita a los estudiantes integrar nueva información en un $27 \%(n=180)$ y se requiere la revisión ortográfica $27 \%$ ( $n=180$ ). En menor medida se solicita eliminar palabras o párrafos ( $23 \%, n=150)$ y unificar la información con un 9\% $(n=60)$. En este caso los escritores experimentados realizan sugerencias de superficie y profundidad en la misma proporción.

En la segunda etapa, los estudiantes pares novatos revisan con una rúbrica el borrador y realizan comentarios abiertos por escrito, sugiriendo modificaciones en estos aspectos: de mejora superficial de ortográfica 34\% ( $n=65)$ y estilo (selección léxica o formal) $33 \%$ ( $n=60)$, y de profundidad, unificación de información, en un 33\% ( $n=60)$. 
Comparativamente frente a los comentarios respecto de la tarea, los estudiantes experimentados sugieran más cambios a nivel de profundidad --dada su experiencia y conocimiento del contenido-- que los novatos de primer año, como explica Baker (2016) respecto de los resultados de su estudio. Los cambios a nivel de superficie constituyen un tipo de pulido del borrador final para desarrollar una mejor técnica de escritura, mientras que los cambios a nivel de significado demuestran un compromiso serio con el texto, la consideración de la audiencia y la auto reflexión.

El impacto de la revisión de los escritores experimentados y de los pares novatos, en la modificación del texto final, de acuerdo con los resultados, de un total de 660 cambios, el 59.1\% ( $n=390)$ concuerda con la revisión del revisor experimentado y solo un $18 \%(n=120)$ de los comentarios de los pares. Lo cual coincide con los resultados del estudio de Paulus (1999) al examinar el efecto de la revisión del maestro en 11 revisiones de estudiantes internacionales en una clase de composición de borradores múltiples, usando protocolos de pensamiento en voz alta, encontró que, de los 843 cambios hechos a ambos borradores, la retroalimentación de los compañeros influyó 13,9\%, mientras que la retroalimentación de los maestros influenció 34,3\%.

Las características de las retroalimentaciones de los revisores experimentados y los pares en el texto final, se muestra en la siguiente figura:

Figura 3. Impacto de la revisión de revisores experimentados y pares en el texto final.

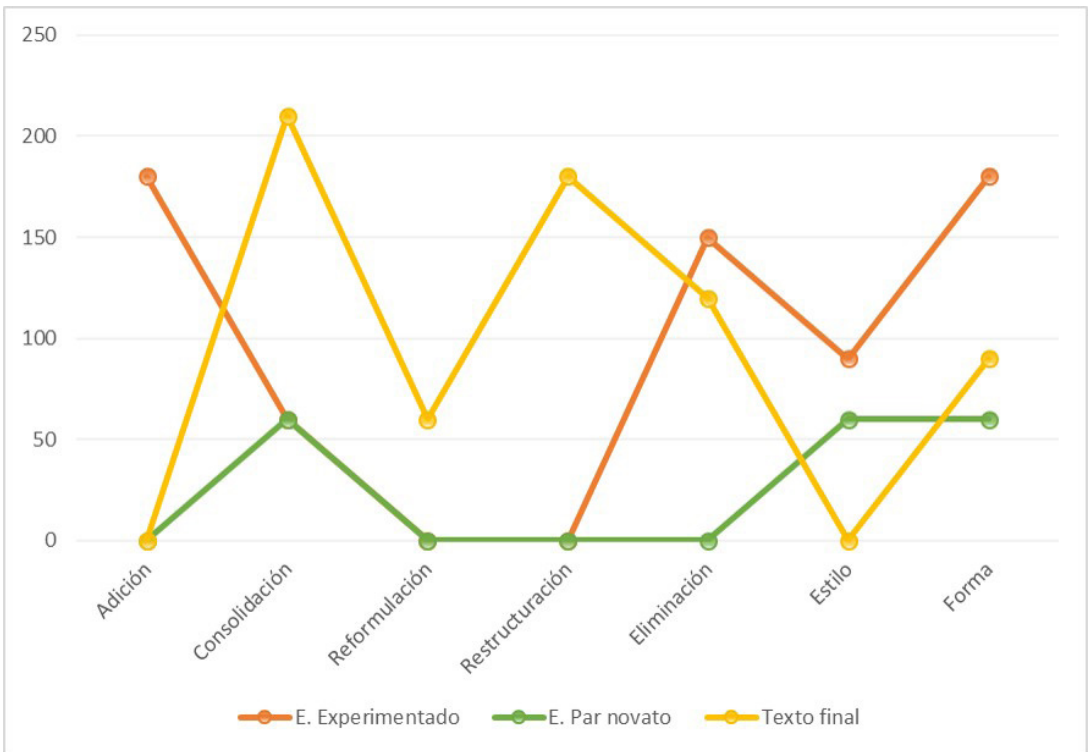

Fuente: Elaboración propia 
A diferencia de los resultados del estudio de Paulus, las dimensiones que se modifican producto de los comentarios son profundas: consolidación y eliminación de información (ver Figura 3), además de, en menor medida, aspectos de forma.

Los cambios realizados al texto final, involucran aspectos de mayor profundidad que los señalados por los escritores expertos y pares novatos: cambios de reformulación y en la reestructuración del texto. En la revisión entre pares, los estudiantes asumen dos roles son a la vez escritores y revisores, lo que se traduce en beneficios para el desempeño de la escritura. Los resultados del estudio de Lundstrom \& Baker's (2009) citados en (Yu y Lee, 2016) sobre los beneficios de realizar comentarios de escritura a los pares, encontraron que quienes tenían menor desarrollo del lenguaje obtuvieron más progresos a nivel global (organización y cohesión) que a nivel local (vocabulario, gramática, entre otros).

Al realizar un análisis de la incidencia de estos comentarios en los cambios finales de profundidad (consolidación, reformulación, reestructuración y forma) se aplica la prueba estadística de chi-cuadrado. De este modo se comprueba la mayor incidencia de los comentarios de los estudiantes expertos (787.7), pero en menor magnitud que la incidencia de los comentarios de los pares (203.3) que resulta menor, aunque el p valor es $<0.00001$. significativo en $\mathrm{p}<.05$ para ambos grupos.

El estudio comentado por Baker (2016) de Li et al. (2010) evidencia la importancia de los comentarios que los revisores pares proporcionan se asocia con un mejor desempeño en los textos finales de los revisores, que la calidad de los comentarios que reciben. Por lo tanto, los revisores que aportan buenos comentarios pueden beneficiarse del proceso de revisión por pares, independientemente de la calidad de los comentarios que reciban como en este estudio, la calidad del texto final no depende solamente de la calidad de los comentarios recibidos. La revisión por pares les permite a los estudiantes ganar autonomía y a ver críticamente su propio trabajo, ayuda a fomentar la autorregulación a medida que aprenden a ver su trabajo desde una perspectiva de los demás.

\section{Escritura reflexiva}

Los procesos de auto-reflexión estimulados por la actividad de revisión de escritura abarcan expresiones de auto-juicio y auto-reacción que intervienen en el proceso de escritura. Una forma de auto-juicio es la autoevaluación, en la que se observan las comparaciones del propio proceso respecto de un estándar (modelo) o al desempeño previo de un compañero. Otra forma de auto-juicio implica la atribución causal, que se refiere a las creencias sobre la causa de los errores o éxitos de uno, frente a los procesos. 
Con cada borrador, se requiere que los estudiantes envíen un comentario reflexivo abierto que responda a lo que habían aprendido al escribir el texto, cuáles habían sido sus dificultades y cómo podrían trabajar para mejorar las áreas de dificultad.

El componente reflexivo del aprendizaje, la enseñanza y la evaluación es un proceso formativo (Andrew, 2005; Lam \& Lee, 2009). Tales estrategias formativas, junto con las intervenciones pedagógicas, la revisión por pares y el trabajo individual y colectivo, dan cohesión y equilibrio sobre el enfoque de escritura. Se presenta a continuación los resultados organizados temáticamente, de acuerdo a la reflexión sobre la actividad de escritura con borradores múltiples y revisión entre pares y de un revisor experimentado.

Figura 4. Temáticas referidas al proceso de escritura mediante revisión de pares novatos y expertos.

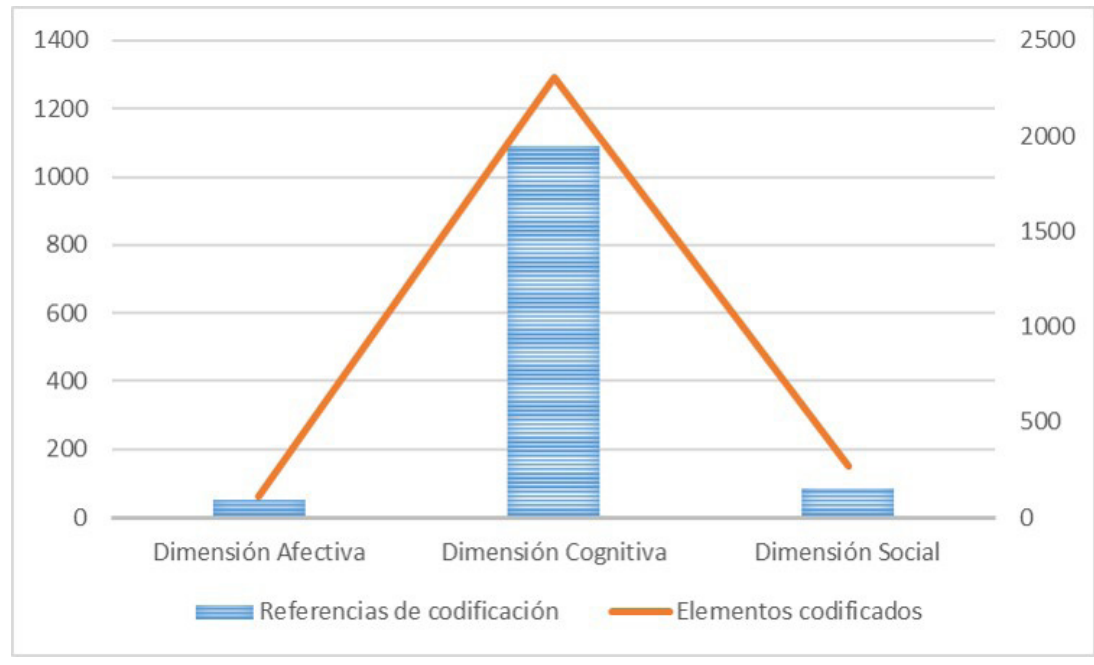

Fuente: Elaboración propia

En la Figura 4, puede apreciarse que los estudiantes de un total de 2190 referencias, un 89\% ( $n=1950)$ hacen mayor alusión a la dimensión cognitiva, específicamente al ámbito metacognitivo respecto al conocimiento de estrategias de aprendizaje y estrategias de escritura, y en menor medida a la autoevaluación de sus escritos. Sólo en un $7 \%(n=150)$ se refieren a la experiencia de intercambio social y en menor medida $4 \%(n=90)$ a al ámbito afectivo.

Los estudiantes de nuestro estudio señalaron que se beneficiaron en gran medida del ambiente de aprendizaje de apoyo, que puso énfasis en la retroalimentación continua de distintos actores, la revisión de los pares y el diálogo respecto de los escritos. Tal ambiente de aprendizaje está en gran contraste con la evaluación de la escritura tradicional, donde la escritura es cronometrada, se realiza en una sola etapa y en la 
que los estudiantes y el trabajo del docente ocurren en aislamiento, como señala uno de nuestros estudiantes:

"Es importante que las estrategias formativas como la revisión por pares y los diálogos sean características regulares del aula de escritura, para que los estudiantes puedan ser apoyados en su proceso de aprendizaje porque antes, hemos escrito muy apresuradamente y cuando el profe nos devolvía los textos revisados, recibíamos algunos breves comentarios. A diferencia de ahora, con la retroalimentación de los facilitadores y los comentarios de los compañeros, me siento mucho más seguro para la revisión de mis borradores".

\section{Reflexiones finales}

Entre los principales resultados de este estudio emergen dos temas: el valor de la revisión de los pares novatos y la importancia de la escritura reflexiva para mejorar la escritura académica.

En general puede señalarse que los estudiantes toman conciencia de su progreso tanto en la escritura académica (referencia, estructuración de párrafos, adición de información, parafraseo y síntesis) como en la adopción de estrategias de escritura (planificación, redacción, corrección de borradores, reflexión respecto de la retroalimentación entre compañeros).

El análisis de las modificaciones de los borradores revela una nueva comprensión de los estudiantes de la edición de sus propios textos y de los de sus compañeros: al examinar la estructura global del texto en lugar de sólo la gramática y la ortografía. Los estudiantes en sus reflexiones informan cambios en sus percepciones del aprendizaje de la escritura adquieren una sensación de progreso y, por lo tanto, reportan una mayor autoconfianza.

Nuestro estudio confirma que cuando los estudiantes están activamente involucrados en la reflexión sobre las dificultades y los retos involucrados en su aprendizaje, obtienen un aprendizaje más profundo de alfabetización académica y de las estrategias fundamentales para elaborar mejores escritos. Las temáticas que los estudiantes abordan en sus reflexiones pueden en sí mismas haber ayudado a promover el pensamiento, el aprendizaje y la adquisición del lenguaje académico. Se observó evidencia de pensamiento analítico y establecimiento de conexiones simples respecto de la "estructura global" (elisiones y adiciones) en relación con los propósitos y resultados de cada tarea de escritura, aunque la mayoría evidenció aprendizajes en el nivel formal (sintáctico y ortográfico). 
El proceso de reflexión generó un nivel de concientización y pensamiento evaluativo. A través de la reflexión, los estudiantes pudieron dar sentido del proceso de redacción de textos académicos en diversos géneros, toman conciencia de su progreso y, tanto aprendices y escritores experimentados, participan en una conversación acerca de esos progresos en la escritura.

No toda la retroalimentación beneficia el aprendizaje, en la literatura se describen varias condiciones bajo las cuales la retroalimentación tiene una influencia positiva en el aprendizaje y que coinciden con los resultados de este estudio. La retroalimentación debe ser (a) suficiente en frecuencia y detalle; (b) debe ser clara respecto a la identificación del problema c) apropiada para el objetivo de la tarea y sus criterios; (d) apropiada en relación con la concepción del aprendizaje, el conocimiento y el discurso de la disciplina de los estudiantes; (e) suficientemente explicativa y (g) la retroalimentación afectiva es también relevante.

La investigación demuestra constantemente que involucrar a los estudiantes en el proceso de retroalimentación mejora la calidad de los textos finales. En parte dado que los estudiantes integran la retroalimentación en sus revisiones y porque valoran que otros verán su trabajo. Aunque también hay evidencia significativa, de que la revisión por pares proporciona beneficios significativos para quienes participan a la vez como escritores y revisores. Además, del impacto en el desarrollo de la escritura, es relevante destacar el fortalecimiento de las estrategias de autorregulación descritas en el proceso reflexivo. De aquí que resulte sugerible incorporar este tipo de actividad como parte del proceso de aprendizaje de la escritura académica.

\section{Referencias}

Baker, K. M. (2016). Peer review as a strategy for improving students' writing process. Active Learning in Higher Education, 17 (3), 179-192. DOI: 10.1177/1469787416654794.

Barton, D.; Ivanič, R.; Appleby, Y; \& Hodge, R. y Tusting, K. (2012). Literacy, lives and learning (Literacy, Lives and Learning). [Disponible en línea en https://www.scopus.com/inward/record.uri?eid=2-s2.084917176285\&doi=10.4324\%2f9780203608302\&partnerlD=40\&md5=9d9dfc7ded96e59732ef30 f650ecdf77].

Cho, K. y MacArthur, C. (2010). Student revision with peer and expert reviewing. En: Learning and Instruction 20 (4), pág. 328-338. DOI: 10.1016/j.learninstruc.2009.08.006.

Cho, K. y MacArthur, C. (2011). Learning by reviewing. Journal of Educational Psychology, 103 (1), 73-84. DOI: 10.1037/a0021950.

Clark, I. (2012). Formative Assessment: Assessment Is for Self-regulated Learning. Educational Psychology Review, 24 (2), 205-249. DOl: 10.1007/s10648-011-9191-6.

Dochy, F.; Segers, M. y Sluijsmans, D. (1999). The use of self-, peer and co-assessment in higher education: A review. Studies in Higher Education, 24 (3), 331-350. DOI: 10.1080/03075079912331379935. 
Gielen, S., Peeters, E., Dochy, F., Onghena, P., y Struyven, K (2010). Improving the effectiveness of peer feedback for learning. Learning and Instruction, 20 (4), 304-315. DOI: 10.1016/j. learninstruc.2009.08.007.

Hattie, J. y Timperley, H. (2007). The Power of Feedback. Review of Educational Research, 77 (1), 81-112. DOI: 10.3102/003465430298487.

Huang, S. C. (2018). A GEARed peer feedback model and implications for learning facilitation. Assessment \& Evaluation in Higher Education, 43 (7), 1194-1210. DOI: 10.1080/02602938.2018.1439881.

Hyland, F. (2000). ESL writers and feedback: Giving more autonomy to students. Language Teaching Research, 4 (1), 33-54. DOI: 10.1177/136216880000400103.

Lachner, A.; Backfisch, I. y Nuckles, M. (2018). Does the accuracy matter? Accurate concept map feedback helps students improve the cohesion of their explanations. Education Tech Research and Development, 66 (5), 1051-1067. DOI: 10.1007/s11423-018-9571-4.

LeMahieu, P., Eresh, J. y Wallace, R. (1992). Using student portfolios for a public accounting. School Administrator, 49 (11), 8-13.

Luaces, O.; Diez, J. y Bahamonde, A. (2018). A peer assessment method to provide feedback, consistent grading and reduce students' burden in massive teaching settings. Computers \& Education, 126 (1), 283-295. DOI: 10.1016/j.compedu.2018.07.016.

Lundstrom, K. y Baker, W. (2009). To give is better than to receive: The benefits of peer review to the reviewer's own writing. Journal of Second Language Writing, 18 (1), 30-43. DOI: 10.1016/j. jslw.2008.06.002.

Mandala, M.; Schunn, C.; Dow, S.; Goldberg, M.; Pearlman, J.; Clark, W. \& Mena, I. (2018). Impact of Collaborative Team Peer Review on the Quality of Feedback in Engineering Design Projects. International Journal of Engineering Education, 34 (4), 1299-1313.

Mendonça, C. O; \& Johnson, K. E. (1994). Peer Review Negotiations: Revision Activities in ESL Writing Instruction. TESOL Quarterly, 28 (4), 745. DOI: 10.2307/3587558.

Min, Hui-Tzu (2005). Training students to become successful peer reviewers. System, 33 (2), 293-308. DOI: 10.1016/j.system.2004.11.003.

Philippakos, Z. A; \& MacArthur, C. A. (2016). The Effects of Giving Feedback on the Persuasive Writing of Fourth- and Fifth-Grade Students. Reading Research Quarterly, 51 (4), 419-433. DOI: 10.1002/ rrq.149.

Semke, H. D. (1984). Effects of the Red Pen. Foreign Language Annals, 17 (3), 195-202. DOI: 10.1111/ j.1944-9720.1984.tb01727.x.

Shah, J.; Rajgor, D. \& Pietrobon, R. (2010). More about the Teaching of Scientific Writing Reply. Academic Medicine, 85 (1), 4-5. DOI: 10.1097/ACM.0b013e3181c48533.

Sommers, N. (2006). Across the drafts. College Composition and Communication, 58 (2), 248-257.

Sluijsmans, D. M. A.; Brand-Gruwel, S.; van Merriënboer, J.J \& Martens, R. L. (2004). Training Teachers in Peer-Assessment Skills: Effects on Performance and Perceptions. Innovations in Education and Teaching International, 41 (1), 59-78. 
Tsui, A.B.M. \& Ng, M. (2000) Does Secondary L2 Writers Benefit from Peer Comments? Journal of Second Language Writing, 9 (1), 147-170. http://dx.doi.org/10.1016/S1060-3743(00)00022-9.

Yu, S. L., \& Lee, I (2016). Peer feedback in second language writing (2005-2014). Language Teaching, 49 (4), 461-493. DOI: 10.1017/s0261444816000161.

Zhang, Shuqiang (1995). Reexamining the affective advantage of peer feedback in the ESL writing class. Journal of Second Language Writing, 4 (3), 209-222. DOI: 10.1016/1060-3743(95)90010-1.

Zhu, Q. Y. (2018). Dialogue within peer feedback processes. Clarification and negotiation of meaning. Higher Education Research \& Development, 37 (4), 883-897. DOI: 10.1080/07294360.2018.1446417.

Yudi Herrera-Núñez

Doctora en en Filología Hispánica. Docente de la Facultad de la educación de la Universidad de Playa Ancha en Chile. Email: yherreran@gmail.com ORCID: https://orcid.org/0000-0003-1406-6089

\section{Correspondência}

Yudi Herrera-Núñez

Facultad de la educación de la Universidad de Playa Ancha

Playa Ancha 850, Valparaíso

Data de submissão: Dezembro 2019

Data de valiação: Fevereiro 2020

Dara de publicação: Novembro 2020 